\title{
The Downward Causality and the Hard Problem of Consciousness or Why Computer Programs Do not Work in the Dark
}

\author{
Alexander Boldachev \\ St. Petersburg, Russia \\ e-mail: alexander@boldachev.com
}

\begin{abstract}
:
Any low-level processes, the sequence of chemical interactions in a living cell, muscle cellular activity, processor commands or neuron interaction, is possible only if there is a downward causality, only due to uniting and controlling power of the highest level. Therefore, there is no special "hard problem of consciousness", i.e. the problem of relation of ostensibly purely biological materiality and non-causal mentality - we have only the single philosophical problem of relation between the upward and downward causalities, the problem of interrelation between hierarchic levels of existence. It is necessary to conclude that the problem of determinacy of chemical processes by the biological ones and the problem of neuron interactions caused by consciousness are of one nature and must have one solution.
\end{abstract}

Keywords: consciousness, hard problem, downward causality.

The hard problem of consciousness was formulated by David Chalmers as a necessity to complement human brain functioning with a conscious experience and subjective mental pictures. ("The really hard problem of consciousness is the problem of experience" [1, p. 201]). This is definitely a stumbling rock for any theories of consciousness, based on the conception of causal closure of the physical: if brain can process all information, transform all the incoming signals into actions without any subjective feelings, then for what brain needs consciousness at all? Let us try to analyze the problem, using the example of computer analogy, i.e. let us focus our attention on a classical Chalmers' question: "why doesn't all this information-processing go on 'in the dark'?" [1, p. 203], in relation to a computer.

At the first glance, this question, when asked about computer functioning, seems just trivial and good-for-nothing. Any "dummy" can understand that while, for example, converting a video file we can turn off the screen, PC-speakers, printer and after this a computer will successfully complete an information processing "in the dark" paying no attention to our actions. And at this point we can say that images on the screen (for example, a line which shows us the status of a process) are nothing more than "epiphenomena" - only the "accompaniment" (in Chalmers' terms) of a real information processing. There seems to be a complete analogy between the information processes in our brain and the mental picture in our consciousness on one hand and the information processing and its display on the screen on the other hand.

But something compels us to resist the temptation of such an easy solution. There is something wrong with this analogy. We understand that a computer, which works only for itself, is a senseless thing, a thing-in-itself. We realize that everything we can interpret as an external "mental" manifestations of computer functioning: a video edit, a photo correction, a computer 
game, etc. - all this makes computer useful. In other words, the picture on the screen is not an "accompaniment" to executed code, but this code has sense only when it is mentally determined, when it performs a certain "higher" function.

And this conclusion about the priority of "the mental", "the higher" in computer over its "lower" information processing becomes more obvious, if we focus our attention at programming. Let us ask the question: from where comes the lowest code level? It is clear, that it is created by a programmer specially and purposefully for providing the "higher" functions. And after that the original question gains the new sense: why a processor does not work "in the dark"? Or, to be more precise, it becomes obvious, that the question is senseless - if there was no original "mental" light, if the "higher" sense of program's work was not formulated, there would be no program.

Of course, the program itself sometimes can work "in the dark". Moreover, there is no necessity to bring to light (to display on the screen) all cycles of array sorting and database queries. Of course, a program is largely autonomous - it does not need a picture on the screen, it does not need to know, what data it works with to perform its function - it needs only some instructions - to take a byte from here, to add one bit, to put it there. But without an external manifestation, without its "higher" sense a program could not come to light, or, to be more precise to flash itself in the "darkness" of computer memory.

After this computer analogy is explicated Chalmers' question ("why doesn't all this information-processing go on 'in the dark'?") asked with the reference to consciousness seems not as sensible as before. Here one is tempted to clarify a point by asking: from where do these "information processes" come in this darkness. Of course, if we are interested only in reactions of a zombie-machine to external signals, the description at the level of "lower informational processes" will surely be enough for us. However, if we face the problem of explaining the nature of reasonable thinking, ethic and aesthetic perception, how can we ask such a stupid question: why all this is in the light? That is because in the dark, at the physiological level there is nothing for which, in fact, they are meant. As we cannot see a text or a picture in a program code, we cannot find any thoughts or emotions at the level of neuron interaction. So we can consider as more sensible the thesis that the lowest procedural level only provides the existence of a mental reality, which is goalsetting for it. The functional requirement list makes the activity of a programmer sensible in the same way. In other words, neither a computer, nor brain can exist without a "mental light" - the "light" precedes a "hardware" and a "hardware" is created for it. Here we deal with a downward causality, not with a linear one.

Naturally, some doubt arises as to the adequacy of given analogy: a computer has no consciousness. But here it is noteworthy that the only difference between a man and a computer is that in the first one (in a man) the mental and the procedural are combined in one "device" and in the second one (in a computer) the "dark" and the "light" levels are distributed in time and space. The whole sense of the computer analogy is that the lowest level of a computer, which seems to be able to work "in the dark", not only can't do it, but it would not appear at all without a "light" level of programmers and users. In other words, the mental levels of both a man and a computer are necessary and fundamental. They form the lowest procedural level and make it sensible. In other words, the problem is not whether a computer has consciousness or not, but in the fact that no "dark" procedurality is possible and sensible without a mental level ("inner" or "external" one).

At this point, we face the main problem - from where comes at the mental level the "requirement list", according to which programs of "information processes" are created in the head of a man? The problem is interesting and really hard. However, in spite of its difficulty, it is far more sensible than a search for aesthetic emotions at the level of neuron interaction. The answer to this question really promises the productive solutions of consciousness problems, unlike the attempts to reveal how the philosophical ideas "look" at the level of metabolite interchange between neurons.

But let us return to the "hard problem of consciousness" and try to reformulate another Chalmers' question: "why is the performance of these functions [perceptual discrimination, 
categorization, internal access, verbal report] accompanied by experience?" [1, p. 204]. Let us say it like this: why chemical reactions are accompanied by mitosis? Or at the physiological and behavioral level: why running of a cheetah is accompanied by the mental action of a "pursuit" and running of a fallow deer is accompanied by an action of "escaping"? We can formulate this question in relation to a computer: why the accomplishment of a command sequence is accompanied by firing of the game characters on the screen?

And at this point, the absurdity of original question becomes obvious. Chalmers' question tacitly implies that the listed low-level processes (chemical reactions, muscle actions, work of a processor, neuron interaction) can proceed per se, independently of higher properties, allegedly only accompanying. But it is clear, that there is no such purely chemical reaction as "mitosis" - it is just stupid to say that a chemical process is accompanied by mitosis. There can be no just running it is always mentally motivated and determined by a higher aim in relation to physiology: its beginning and end is not determined at the cellular level of muscles. It is senseless to speak about a program code, which is not written for realization of certain high-level tasks - video processing or spell checking.

In actual fact, it is impossible to explain mitosis, hunt of a cheetah, changing pictures on the screen only as interpretations, as epiphenomena of self-sufficient, autonomous low-level processes. The opposite is obvious: the sequences of elementary chemical reactions, muscle actions and processor commands are determined by these very epiphenomena - biological life of a cell, psychic activity of higher Metazoans, the will of a programmer. Literally, every next chemical reaction in mitosis is not determined by a previous reaction. All chemical reaction together implement the mitosis, but these elementary interactions do not comprise a single chemical process, united selfsufficient chemical reaction - here we have many simultaneous processes united by a non-chemical causality. Just like this a muscle contraction of a cheetah's hind leg is not determined by its previous contractions - we cannot speak about any causal closure at physiological level, for which psychic is only an external optional form. We have just an opposite picture - all physiological processes are subject to the highest psychic goal-setting.

Here the question suggests itself: why when formulating "the hard problem of consciousness" the neuron substrate of human brains is placed in the causally closed physical world? Chalmers puts the problem of casual dependence of consciousness and neurophysiologic processes as if the problem of determination of life, its reduction to chemical processes and further to physical interaction has already been solved. After all, such "hard problem" can also be formulated with respect to interrelation of biologic metabolism and organic synthesis reactions: why the causally closed chemical processes are accompanied by the "biological experience"? In other words, if it is acceptable to speak about the causal closure of the physical, then only at chemical level and lower levels - and according to the "hard problem" logic, life should be interpreted only as an epiphenomenon.

Why at least some hints of solution of consciousness problem along the lines of epiphenomenalism or psychophysical parallelism are possible at all? Problems of relation between low-level and high-level properties, which were already formulated for relation between the chemical and the biologic, the physiologic and the psychic, definitely indicate the ontological inadequacy of such approach. Where can we see the difference between the relation, on the one hand, of muscle cells and psyche, and, on the other hand, of neurons and consciousness? Why it is so obvious to us that the state of one kind of cells (muscle cells) is determined by the high-level causality - the mental actions like "pursuit" or "escaping" - and the states of another kind of cells (neurons) determine each other like gears in a clock? Why do we understand that life is something more than a chemical reaction, and at the same time accept that the consciousness is only a name for a self-sufficient sequence of neuron interaction? Of course, even running action is a sequence of cellular activity, but the problem is not in realization, it is in causality.

So, the simple analysis of empiric facts makes us come to the following conclusion: any low-level procedurality - the sequence of chemical interactions in a living cell, muscle cellular 
activity, processor commands or neuron interaction is possible only if there is a downward causality, only due to uniting and controlling power of the highest level (see [3, pp. 86-101]).

Therefore, there is no special "hard problem of consciousness", i.e. the problem of relation of ostensibly purely biological materiality and non-causal mentality - we have only the single philosophical problem of relation between the upward and downward causalities, the problem of interrelation between hierarchic levels of existence. It is necessary to conclude, that the problem of determinancy of chemical processes by the biological ones and the problem of neuron interactions caused by consciousness are of one nature and must have one solution.

\section{References}

1. Chalmers, D.J. Facing up to the problem of consciousness, Journal of Consciousness Studies, 2 (3), 1995, pp. 200-219.

2. Chalmers D.J. The Conscious Mind. In Search of a Fundamental Theory. N. Y.: Oxford, 1996.

3. Boldachev, A.V. Temporality and the philosophy of absolute relativism, Moscow: Lenand, 2011 (in Russian). 